\title{
The Unmet Needs of African American Women with Breast Cancer
}

\author{
Marlene M. von Friederichs-Fitzwater ${ }^{1}$, Reverend Tammie Denyse ${ }^{2}$ \\ ${ }^{1}$ University of California Davis, School of Medicine, Sacramento, USA \\ ${ }^{2}$ Carrie's TOUCH, Sacramento, USA \\ Email: marlene.vonfriederichs-fitzwater@ucdmc.ucdavis.edu
}

Received February 28, 2012; revised March 27, 2012; accepted April 5, 2012

\begin{abstract}
Breast cancer is the most common cancer in women and treatment includes various combinations of surgery, chemotherapy, and/or hormone therapy. The multimodal treatment of breast cancer improves survival outcomes, but it also contributes to a prolonged period of medical intervention with associated physical and emotional consequences. However, we know less about the specific clinical experiences of African American (AA) women during treatment for breast cancer and in survivorship. The studies that have addressed the issue of breast cancer in AA women have focused primarily on early detection and epidemiological variables such a screening, mortality and staging at diagnosis. In our study, in-depth semi-structured phone interviews were conducted to explore the clinical experiences, concerns and needs of AA women who had survived breast cancer. The AA women reported that their physicians did not provide adequate disease and treatment information; did not discuss clinical trials with them; and did not offer access to support services.
\end{abstract}

Keywords: Breast Cancer; African American Women; Treatment; Survivorship

\section{Introduction}

Breast cancer is the most common cancer in women and treatment includes various combinations of surgery, chemotherapy, and/or hormone therapy [1]. The multimodal treatment of breast cancer improves survival outcomes, but it also contributes to a prolonged period of medical intervention with associated physical and emotional consequences [1]. Research has demonstrated that women with disease-free survival beyond five years often experience psychological distress, concerns about rehabilitation, and employment difficulties as a result of the diagnosis [2-5].

However, we know less about the specific experiences of African American (AA) women during treatment for breast cancer and in survivorship. The studies that have addressed the issue of breast cancer in AA women have focused primarily on early detection and epidemiological variables such a screening, mortality and staging at diagnosis [6-9]. The few studies from the 1990s that addressed psychosocial issues among breast cancer patients found that AA women experience a high degree of fatalism, lack of knowledge, lack of trust in the medical system and concerns about social and emotional support [10,11]. A study by Ashing-Giwa and Ganz [12] used three qualitative methods to obtain more information about the breast cancer experiences of AA women find- ing a lack of adequate social support and a need of AA women for advocacy and support during their breast cancer diagnosis and treatment. The women in their study also lacked specific information about their diagnosis and treatment options.

In our study, in-depth semi-structured phone interviews were conducted to explore the experiences, concerns and needs of AA women who had survived breast cancer.

\section{Method}

A mixed-methods approach (qualitative and quantitative) was used in the study. The participants were recruited from breast cancer support groups for AA women, survivorship events, community groups and breast cancer support agencies. A semi-structured instrument was developed that included open and close-ended questions on demographics, diagnosis, treatments, clinical trials, information seeking, support services, lingering effects of treatments, insurance coverage and employment history. The instrument was field tested with five AA breast cancer survivors. AA breast cancer survivors were called to verify their eligibility and willingness to be interviewed and consented; telephone interviews were then scheduled and conducted with those who agreed to participate. All identifying information was removed from the data and stored separately. 


\section{Results}

A total of 137 participants completed the survey, with the average age of 52 years at time of diagnosis; $49 \%$ were not married; and 56\% had graduated high school or completed a two-year degree, $30 \%$ had completed four years of college. Seventy percent had been diagnosed with breast cancer within the past five years; 30\% had been diagnosed prior to 2006; and six of the participants had experienced a recurrence of cancer since their initial diagnosis.

\subsection{Treatment Experiences}

Participants reported waiting from 0 - 60 days from diagnosis to the beginning of treatment with $30 \%$ reporting 0 - 15 days, 43\% reporting waiting 16 - 30 days, 16\% waiting 31 - 45 days and 5\% waiting 46 - 60 days. Five percent did not respond to the question. Participants said they believed that beginning treatment promptly after diagnosis was important and the majority indicated that they were comfortable with the length of time they experienced. The women (22\%) who reported waiting between 31 and 60 days before beginning treatment were not satisfied with the wait time; they reported a desire for some type of support during the time between diagnosis and the beginning of treatment. See Figure 1.

Sixty percent of the women surveyed had surgery (mastectomy single breast or mastectomy double breast; lumpectomy) frequently followed with radiation and/or chemotherapy. None of the women had breast reconstruction at the time of the initial surgery and one woman had breast reconstruction after her initial surgery. Four women reported "Other" treatments along with surgery (e.g., hormonal treatment) (See Figure 2 for types of treatment received.).

The majority of women (78\%) received the treatment (s) recommended by their doctors (oncologist, surgeon); $70 \%$ reported that the treatment was what they would have chosen while 19\% said they weren't sure and had wanted more information; 5\% would not have chosen the treatments they had.

Eighty-one percent of the participants did not receive any complementary medicine (e.g., acupuncture, acupressure, massage, homeopathy, herbal remedies, etc.), but said they would have liked to have information about such options to help with side effects and overall wellbeing.

\subsection{Information Needs}

Ninety percent of the women were satisfied with their treatment, but $24 \%$ were not satisfied with the information they received from their doctors on breast cancer and treatment options. The women who were not satisfied with their treatment or with the amount of information
Time Lapsed Between Initial Diagnosis \& Beginning of Treatment

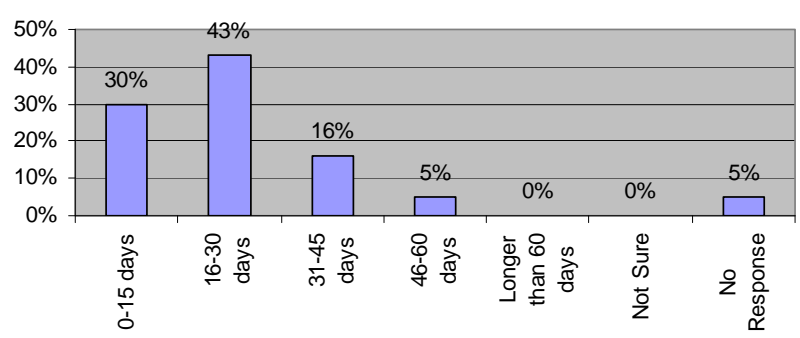

Figure 1. Time lapse between diagnosis and treatment.

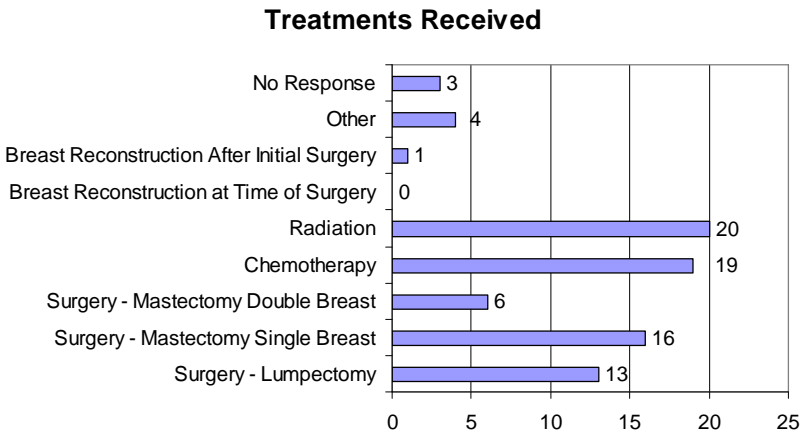

Figure 2. Treatments received.

received reported that they wanted more aggressive treatment, a second opinion and more information specific to AA women (e.g., how they might respond to treatment, side effects they might expect, etc.).

The majority of participants (60\%) did seek information on their own with $45 \%$ of them using the Internet; 9\% using print materials and another 9\% getting second opinions. Almost half of the women (43\%) said their doctor or other healthcare provider did not provide information about support services while they were going through treatment.

\subsection{Support Services}

Sixty-two percent of the women reported wanting support services such as opportunities to have one-on-one discussions with a woman who was a breast cancer survivor such as a peer navigator to help them understand what to expect and provide accurate and credible information. They also wanted a list of support groups and someone from a support group to meet with them before leaving the hospital. Some participants wanted access to complementary services such as massage and yoga. When asked what support services they actually experienced, the majority said "none," with one woman reporting that she went to an American Cancer Society Look Good, Feel Better class and another stating she had met with a therapist. Of those who responded to the question, 24\% were not at all satisfied they received dur- 
ing treatment. With respect to the support services they received after treatment, $22 \%$ were not at all satisfied.

\subsection{Clinical Trials}

AAs tend to be underrepresented in clinical trials and $78 \%$ of the women in this study reported that their doctors did not talk with them about enrolling in a clinical trial or even discuss the option. The majority of women (78\%) said they would have liked to know about clinical trials and if appropriate, would have enrolled.

\subsection{General Health Status}

Over half of the participants reported that on average their health was about the same as it was a year ago and $68 \%$ said they did not have any current health conditions that limited their ability to work or carry out daily activities. However, 8\% reported "lingering side effects" such as pain and lymphoedema; and $11 \%$ said they suffered from insomnia and fatigue since treatment. Twenty-two percent have other chronic diseases such as diabetes, hypertension and arthritis.

\subsection{Lingering Effects}

Participants reported such lingering effects as fatigue, anxiety, depression, stress, new health problems ("heart problems," "hypertension," "joint pain”), recurrence or a new cancer type diagnosis and neuropathy. They also reported not being satisfied with the results of surgery and swelling around chest area. Ninety-two percent said they had an ongoing fear of recurrence of cancer and were more aware of their bodies than before their diagnosis and treatments.

\subsection{Insurance and Employment}

Eighty-six percent of the women had some form of insurance during treatment and continued to have coverage after treatment. They reported that having adequate insurance was a major concern, but they were able to utilize private or public coverage.

When asked if they had trouble taking time off work during treatments (if they worked), 27\% said they did have problems and one woman who reported not having problems taking time off also reported that her position was filled during her absence. Thirty-five percent did not answer the question and $27 \%$ said they didn't work outside the home.

While the participants did not have to worry about coverage for their treatments, those women who worked outside the home did struggle with work-related problems during treatment and when they returned to work (if their former jobs were available). This caused additional stress for women who had to deal with co-workers and supervisors who treated them differently upon their return, and for those who had to seek new jobs. Participants reported being uncertain about to how to communicate with supervisors or colleagues about their cancer experience.

\subsection{Specific Concerns as AA Women with Breast Cancer}

When asked specifically about they thought their experiences were different from non-AA women diagnosed with breast cancer, the majority of participants reported the following concerns relevant to the quality of their care:

- Uncertainty about whether or not healthcare providers understand the possible differences in response to treatments of AA women diagnosed with breast cancer;

- Potential for poor quality care or lack of adjuvant therapies because of race or socioeconomic circumstances;

- Perceptions that information was being withheld because of their race; and;

- Unavailability of resources and support groups specifically for AA women diagnosed with breast cancer.

\section{Discussion}

While the sample size for this study was small, the data obtained adds new insights into understanding AA women's experiences with breast from diagnosis through short-term and long-term survivorship and will help to guide the development and delivery of patient education and support services to better service AA women.

We learned that the time between initial diagnosis and the beginning of treatment can be from 0 - 60 days with $21 \%$ of the women waiting 31 - 60 days. Studies indicate the highest levels of anxiety occur between diagnosis and the beginning of treatment [13]. The waiting time provides an opportunity to reach out to AA women diagnosed with breast cancer to help them prepare for their first visit with an oncologist or surgeon and their subsequent treatment. Providing one-on-one support during this time by a trained peer or patient navigator, preferably AA, would lay the groundwork for continued support through treatment and fulfill the reported need for more accurate and credible information as well as support. They could also be linked to other resources such as the Breast Cancer Network of Strength and the American Cancer Society during this time as they plan to begin treatment.

Many of the survivors lacked adequate information from their physicians about their diagnosis, treatment and symptom management. Some sought information on their own from the Internet, print materials and getting second 
opinions, but were not confident that it was accurate or credible. This lack of adequate information sometimes resulted in treatment decisions that women later regretted or questioned. Clearly, there is need to provide the amount and level of information required by patients prior to their treatment decision-making and to help patients make sense of the available information.

In addition, the women did not receive information about support services and resources to the extent they desired. With the majority of women single, widowed, separated or divorced, they often did not have adequate family support, but some did report that they received assistance from their churches and friends. Some of the women raised the need for support groups and resources specifically for AA women and reported that they felt uncomfortable in support groups where they were the only AAs. One woman said:

I went to a support group and it was all these white women talking about their families and their jobs. I felt out of place, like a sore thumb. I am not married, don't have family here and I don't work. I never went back.

In addition to the demographic make-up of the support group, some women are comfortable in a group format for support and some are not. For the latter, there need to be options so that women have choices about receiving support (one-on-one support, telephone support, Internet support groups, video-conference support groups, etc.).

Although many factors contribute to poor cancer survival rates among racial and ethnic minorities, healthrelated behaviors such as information-seeking and participation in treatment decision-making, remain a powerful factor in cancer mortality rates among all groups [14]. Additional research is needed to identify behavioral factors associated with poor outcomes in AA cancer patients, particularly in those areas that may be amenable to intervention [14]. According to Pope-Davis \& Coleman, culture reflects a group's or individual's social and physical environment, communication patterns, and shared beliefs, values and traditions, all resulting in common lifestyles, attitudes and behaviors [15].

These same factors can result in insufficient information seeking and acquisition, which can lead to ill-considered decisions about alternative treatment options [16]. Patients who are adequately informed about their illness and treatment options are better able to maintain a sense of control (cite) and to cope with the uncertainty of the illness and its treatments (cite). Twenty-four percent of the women in this study were not satisfied with the information they received on their breast cancer and treatment options and said they did not understand treatment options, did not understand the information provided, were not told about long term side effects, and did not receive information on how medications would effect AA women. They also reported that physicians did not spend enough time talking to them. These are all issues that can be addressed and ameliorated with tailored patient education materials, tools for healthcare providers to assess patient information needs and the use of understanding strategies such as "Repeat-Back" by healthcare providers (doctor asks the patient to repeat back what he/she said).

Enrollment in cancer trials is low for all patient groups. Racial and ethnic minorities, women and the elderly are less likely to enroll in cancer clinical trials than were white, men and younger patients, respectfully [17]. It is well known that minority recruitment into clinical trials faces a problem with rates of participation, ranging from $3 \%$ to $20 \%$ [18]. The proportion of clinical trial participants who are black has also declined in recent years [17].

There are a number of reasons for low enrollment of all patients and some specific reasons for low enrollment of minority patients: setting of care (community clinic, academic institution, etc.); ineligibility of patient for existing trials; lack of available clinical trial for patient's cancer type/stage; patient's health status; and in some states, lack of insurance to cover clinical trial participation. Patients in California are guaranteed coverage for standard of care while participating in appropriate clinical trials, but there are at least two specific reasons for the lack of minority enrollment. Past discussions and research on minority enrollment have highlighted several barriers that are more prevalent among specific minority groups [17]. First, minorities are more likely to express concerns about exploitation, dishonesty regarding risks of experimental treatment, and motivations of researchers [14]. A strong belief that physicians are not strong allies for their AA patients contributes to lack of trust and fear of being lied to/or experimented on [14].

For some this distrust is based at least in part on the abuses of AAs in the Tuskegee experiments. Other studies have suggested that minority patients may be less likely to be offered trial participation [18]. In our study, we found that in the majority of encounters with patients, physicians did not raise the topic. Several of the women indicated they would have liked to discuss the option with their physicians, but didn't because the doctors didn't bring it up.

This might also indicate the usefulness of trained peer navigators being assigned to newly diagnosed patients and available to provide information on clinical trials as well as suggest strategies for discussing them with physicians. It may also be that physicians are more reluctant to discuss clinical trials with minority patients and may appreciate the assistance of a trained peer navigator of the same race/ethnicity and age in doing so.

Fatigue, breast and arm pain, and problems with lymphoedema are the most commonly reported late effects of 
treatment by both younger and older women in previous research [19]. Rather than the need for specific treatment of their symptoms (e.g., fatigue, pain), many women speak of their unmet need to determine whether their symptoms were or were not a sign of recurrence. This study was no exception. One woman said:

I am so tired now, never used to be. I get wore out doing simple things. My grandkids, they don't understand. Why I can't play with them like I used to. It makes me feel like I am a patient, still going through treatment... and it worries me. Does it mean there is cancer somewhere in my body? Is that why I am so tired?

Participants in this study reported issues with returning to work and concerns about the overall quality of their care. The women reported concerns about not receiving the same quality of care and options that their non-AA counterparts might receive. While they could not report specific detailed examples, the concerns were significant to them.

This study has limitations. First, the sample size was small and participants were not selected randomly, thus, may not accurately reflect the experiences of all AA women diagnosed with breast cancer. Because this study was largely a qualitative study, the generalizability of results beyond study participants is not recommended nor is it the intent of qualitative research.

Findings do, however, provide important implications for future research and program development. First, more research regarding the feasibility and efficacy of recruiting and training AA breast cancer survivors as peer navigators (cancer coaches) to work with newly diagnosed AA women immediately following diagnosis. Second, more attention should be paid to ensuring support services are in place that are culturally acceptable and appealing to AA women diagnosed with breast cancer and, then, ensuring that healthcare providers as well as AA patients are informed about their availability. Collaborations with existing community groups, faith-based organizations and AA churches continue to be an overlooked resource for disseminating information to newly diagnosed AA women. With regard to work-related issues, research and programs need to be developed that will make the transition back to work smoother for all cancer patients at all employment levels.

\section{Acknowledgements}

We thank the AA women who participated in this study, the Sutter Foundation and the Breast Cancer Network for Strength for funding support and Carrie's TOUCH, a nonprofit community organization for AA women with breast cancer, for instrument development and recruiting assistance.

\section{REFERENCES}

[1] P. A. Ganz, J. H. Rowland, K. Desmond, B. E. Meyerowitz and G. E. Wyatt, "Life after Breast Cancer: Understanding Women's Health-Related Quality of Life and Sexual Functioning,” Journal of Clinical Oncology, Vol. 16, No. 2, 1998, pp. 501-514.

[2] C. Coscarelli-Schag, P. A. Ganz, M. Polinsky, C. Fred, K. Hirji and L. Peterson, "Characteristics of Women at Risk for Psychosocial Distress in the Year after Breast Cancer,” Journal of Clinical Oncology, Vol. 11, No. 4, 1993, pp. 783-793.

[3] P. A. Ganz, K. Hirji, M. Sim, C. Coscarelli-Schag, C. Fred and M. Polinsky, "Predicting Psychosocial Risk in Patients with Breast Cancer,” Medical Care, Vol. 31, 1993, pp. 419-431. doi:10.1097/00005650-199305000-00004

[4] M. Polinsky, "Functional Status of Long-Term Breast Cancer Survivors: Demonstrating Chronicity,” Health \& Social Work, Vol. 19, No. 3, 1994, pp. 165-173.

[5] J. Rowland and M. Massie, "Psychologic Reaction to Breast Cancer Diagnosis, Treatment and Survival,” In: J. Harris, M. Lippman, M. Morrow and S. Hellman, Eds., Diseases of the Breast, Lippincott-Raven, Philadelphia, 1996, pp. 919-938.

[6] V. Champion, "Development of a Benefits and Barriers Scale for Mammography Utilization,” Cancer Nursing, Vol. 18, No. 1, 1995, pp. 53-59. doi:10.1097/00002820-199502000-00008

[7] C. Lerman, E. Lustbader, B. Rimer, M. Daly, S. Miller, C. Sanda and A. Balshem, "Effects of Individualized Breast Cancer Risk Counseling: A Randomized Trial,” Journal of the National Cancer Institute, Vol. 87, No. 4, 1995, pp. 286-292. doi:10.1093/jnci/87.4.286

[8] E. Long, "Breast Cancer in AA Women: Review of the Literature,” Cancer Nursing, Vol. 16, No. 1, 1993, pp. 124.

[9] J. Moormeier, "Breast Cancer in Black Women,” Annals of Internal Medicine, Vol. 124, 1996, pp. 897-905.

[10] H. Matthews, L. Donald and J. Mitchell, “Coming to Terms with Advanced Breast Cancer: Black Women's Narratives from Eastern North Carolina,” Social Science \& Medicine, Vol. 38, No. 6, 1994, pp. 789-800.

[11] M. Powell, "Social and Psychological Aspects of Breast Cancer in AA Women,” Annals of the New York Academy of Sciences, Vol. 736, 1994, pp. 131-139. doi:10.1111/j.1749-6632.1994.tb12825.x

[12] K. Ashing-Giwa and P. A. Ganz, "Understanding the Breast Cancer Experience of AA Women," Journal of Psychosocial Oncology, Vol. 15, No. 2, 1997, pp. 19-35. doi:10.1300/J077v15n02_02

[13] P. M. Triefm and M. Donohue-Smith, "Counseling Needs of Women with Breast Cancer: What the Women Tell Us,” Journal of Psychosocial Nursing, Vol. 34, 1996, pp. 24-29.

[14] A. K. Matthews, S. A. Sellergren, C. Manfredi and M. Williams, "Factors Influencing Medical Information Seeking among AA Cancer Patients,” Journal of Health 
Communication, Vol. 7, No. 3, 2002, pp. 205-219. doi:10.1080/10810730290088094

[15] D. B. Pope-Davis and H. L. K. Coleman, "Multicultural Counseling Competencies: Assessment, Education and Training, and Supervision," Sage Publications, Thousand Oaks, 1997.

[16] C. Lerman, M. Daly, W. P. Walsh, N. Resch, J. Seay, A. Barsevick, et al., "Communication between Patients with Breast Cancer and Health Care Providers: Determinants and Implications," Cancer, Vol. 72, No. 9, 1993, pp. 2612-2620. doi:10.1002/1097-0142(19931101)72:9<2612::AID-CNC R2820720916>3.0.CO;2-F

[17] V. H. Murthy, H. M. Krumholz and C. P. Gross, "Par- ticipation in Clinical Trials: Race-, Sex- and Age-Based Disparities," The Journal of the American Medical Association, Vol. 291, No. 22, 2004, pp. 2720-2726. doi:10.1001/jama.291.22.2720

[18] L. L. Adams-Campbell, C. A. Ahaghotu, M. Gaskins, F. W. Dawkins, D. Smoot, O. D. Polk, et al., "Enrollment of African Americans onto Clinical Treatment Trials: Study Design Barriers,” Journal of Clinical Oncology, Vol. 22, No. 4, 2004, pp. 730-734. doi:10.1200/JCO.2004.03.160

[19] B. Thewes, P. Butow, A. Girgis and S. Pendlebury, "The Psychosocial Needs of Breast Cancer Survivors: A Qualitative Study of the Shared and Unique Needs of younger Versus Older Survivors,” Psycho-Oncology, Vol. 13, No. 3, 2003, pp. 177-189. doi:10.1002/pon.710 